\title{
Machine Learning Transition Temperatures from 2D Structure
}

\author{
Andrew E. Sifain, ${ }^{\dagger}$ Samuel H. Yalkowsky, ${ }^{\ddagger}$ Betsy M. Rice, ${ }^{\dagger}$ and Brian C. Barnes ${ }^{*} \dagger$ \\ $\dagger C C D C$ U.S. Army Research Laboratory, Aberdeen Proving Ground, MD 21005, USA \\ $\ddagger$ Department of Pharmaceutics, College of Pharmacy, University of Arizona, Tucson, AZ \\ 85721, USA \\ E-mail: brian.c.barnes11.civ@mail.mil
}

\begin{abstract}
A priori knowledge of physicochemical properties such as melting and boiling could expedite materials discovery. However, theoretical modeling from first principles poses a challenge for efficient virtual screening of potential candidates. As an alternative, the tools of data science are becoming increasingly important for exploring chemical datasets and predicting material properties. Herein, we extend a molecular representation, or set of descriptors, first developed for quantitative structure-property relationship modeling by Yalkowsky and coworkers known as the Unified Physicochemical Property Estimation Relationships (UPPER). This molecular representation has groupconstitutive and geometrical descriptors that map to enthalpy and entropy; two thermodynamic quantities that drive thermal phase transitions. We extend the UPPER representation to include additional information about $s p^{2}$-bonded fragments. Additionally, instead of using the UPPER descriptors in a series of thermodynamically-inspired calculations, as per Yalkowsky, we use the descriptors to construct a vector representation for use with machine learning techniques. The concise and easy-to-compute representation, combined with a gradient-boosting decision tree model, provides an appealing
\end{abstract}


framework for predicting experimental transition temperatures in a diverse chemical space. An application to energetic materials shows that the method is predictive, despite a relatively modest energetics reference dataset. We also report competitive results on diverse public datasets of melting points (i.e., OCHEM, Enamine, Bradley, and Bergström) comprised of over 47k structures. Open source software is available at https://github.com/USArmyResearchLab/ARL-UPPER.

\section{Introduction}

Transition temperatures such as melting and boiling point are fundamental thermodynamic properties that influence applications including the design of pharmaceuticals, 1 melt-casted explosives, 2[4] and energy harvesting materials. [56] Discovering materials with acceptable transition temperatures is difficult, in part because they are not known prior to synthesis and measurement. Theoretical prediction of such properties may reduce the chemical space of candidate compounds and expedite discovery. Thus, a great amount of effort has been expended in both high-fidelity physics-based simulations and in regression-based modeling of phase transition temperatures. The main advance in this work is the extension of a set of thermodynamically-inspired descriptors, combined with a gradient-boosting machine learning technique to create a new, accurate, and rapid model for predicting phase transition temperatures.

Atomistic simulations of phase transitions are computationally demanding. Furthermore, in the case of melting, such simulations often require knowledge of crystal structure, $7-10]$ thus limiting their use during materials discovery. An alternative approach is to utilize surrogate models that map descriptors to reference data. Linear regression-based quantitative structure-property relationships (QSPRs) have had success, 11 [16 but by design cannot discover a new, nonlinear mapping between descriptors and melting point. Nonlinearity may be provided in advance using knowledge of relevant physics and chemistry. Nonlinear machine learning (ML) algorithms have no such requirement for prior knowledge of nonlinear relation- 
ships $17 \sqrt[20]{20}$ and when carefully applied, possess other advantages such as transferability to species outside of the reference dataset and computational efficiency. 21 23] An accurate ML model may help identify target compounds and circumvent expensive atomistic simulations.

ML is becoming an indispensable and versatile tool in the chemical sciences 24 with application to molecular properties, 25, s1] spectroscopy, $, 32,36$ and chemical synthesis. $37+40$ Model performance depends concomitantly on the learning algorithm, the quality of the reference training set, and the input representation of the chemical system. 4147 The focus of this paper is on the combination of a molecular representation, derived from a microscopic basis, with a gradient-boosting algorithm in order to predict macroscopic properties such as melting and boiling point.

The molecular representation reflects the level of chemical resolution needed for predicting the target property. $\stackrel{48[49}{ }$ For quantum molecular properties, geometry and atom type are typically chosen as input because even the slightest changes in geometry can affect the wavefunction and its observables. $[50$ 53] On the other hand, macroscopic properties are more robust to higher-level or coarse-gained descriptors. $\underline{54}$ [58 In the case of melting, for example, a molecular crystal may be identified by descriptors $[59] 62]$ derived from its repeating structural unit. ${ }^{20}$ The mapping of a multi-molecule process from a single molecule is ambiguous however, thus emphasizing the importance of suitable descriptors.

The proposed representation is both inspired by and is an extension of the UPPER method of Yalkowsky and coworkers. $\frac{63}{6 P P E R}$, which stands for Unified Physicochemical Property Estimation Relationships, is a comprehensive QSPR based on intuitive and thermodynamic relationships relating phase transition properties to one another including transition enthalpies, entropies, and temperatures. The method extends to other properties including heat of sublimation and solubility. UPPER's descriptors are derived purely from $2 \mathrm{D}$ structure (i.e., Simplified Molecular-Input Line-Entry System or SMILES [64), allowing for simple and fast property prediction following regression with a learning algorithm. While it is a challenge to train models to limited and noisy experimental data, we find that this molecu- 
lar representation combined with gradient boosting, ${ }^{65}$ provides an appealing framework for accurately predicting experimental transition temperatures in a diverse chemical space. Our software is freely available at https://github.com/USArmyResearchLab/ARL-UPPER.

\section{Methods}

We provide an overview of the UPPER method for transition properties and the underlying descriptors that will be supplied as input for ML. The addition of heat to a thermodynamic system increases its temperature. When a first-order phase transition occurs, the temperature levels off, remaining constant even as the system continues absorbing heat. Intermolecular binding forces are overcome as heat converts the state of the system from solid to liquid or liquid to gas. When the two phases of matter are in equilibrium with one another, the Gibbs energy is $\Delta G_{t r}=0$. The first-order transition temperature can therefore be written as

$$
T_{t r}=\frac{\Delta H_{t r}}{\Delta S_{t r}}
$$

where $\Delta H_{t r}$ and $\Delta S_{t r}$ are enthalpy and entropy of transition, respectively. Here, $\Delta H_{t r}$ is the amount of heat absorbed per mole for the transition to take place, while $\Delta S_{t r}$ is the

change in the system's entropy. UPPER defines analytical forms for $\Delta H_{t r}$ and $\Delta S_{t r}$ with parameters determined using separate linear regression analysis of composition for $\Delta H_{t r}$ and geometry for $\Delta S_{t r}$.

\section{Group-Constitutive Descriptors}

Within UPPER, enthalpy is computed as a group-constitutive property,

$$
\Delta H_{t r}=\sum_{i} p_{i} n_{i}
$$


where $p_{i}$ is the contribution of the $i$-th fragment and $n_{i}$ is the number of $i$ fragments in the molecule. Fragmentation is based on the scheme proposed in Ref. [66], where each fragment consists of the least number of atoms (including all carbons, hydrogens, heteroatoms, and nonbonded electrons) that are not separated by an isolating carbon. An isolating carbon is a carbon that is not doubly or triply bonded to a heteroatom. Such carbons and their attached hydrogens are considered hydrophobic fragments and the remaining groups of atoms are polar fragments. Fragments are represented by their SMARTS (SMiles ARbitrary Target Specification) strings and subsequently assigned to an environmental group (Fig. 1). Environmental groups (Table 1) reflect interactions such as the connectivity and hybridization that each fragment has with its neighboring fragments. New environmental groups added in this work include YYY, YYYY, YYZ, and YYYZ. These groups generalize the featurization to fragments with several $s p^{2}$-hybridized bonds. Throughout this work, we use our extended set of descriptors as described in Table 1.

Table 1: Environmental groups.

\begin{tabular}{c||c} 
Group & Description \\
\hline \hline $\mathrm{X}$ & Group bonded to only $\mathrm{sp}^{3}$ atoms \\
$\mathrm{Y}$ & Group singly bonded to $1 \mathrm{sp}^{2}$ atom \\
YY & Group bonded to $2 \mathrm{sp}^{2}$ atoms \\
YYY & Group bonded to $3 \mathrm{sp}^{2}$ atoms \\
YYYY & Group bonded to $4 \mathrm{sp}^{2}$ atoms \\
Z & Group bonded to $1 \mathrm{sp}$ atom \\
YZ & Y and Z group \\
YYZ & YY and Z group \\
YYYZ & YYY and Z group \\
RG & Group within an aliphatic ring \\
FU & Aliphatic bridge-head group \\
AR & Group within an aromatic ring \\
BR2 & Aromatic carbon shared by 2 rings \\
BR3 & Aromatic carbon shared by 3 rings \\
BIP & Central carbon in biphenyl substructure
\end{tabular}




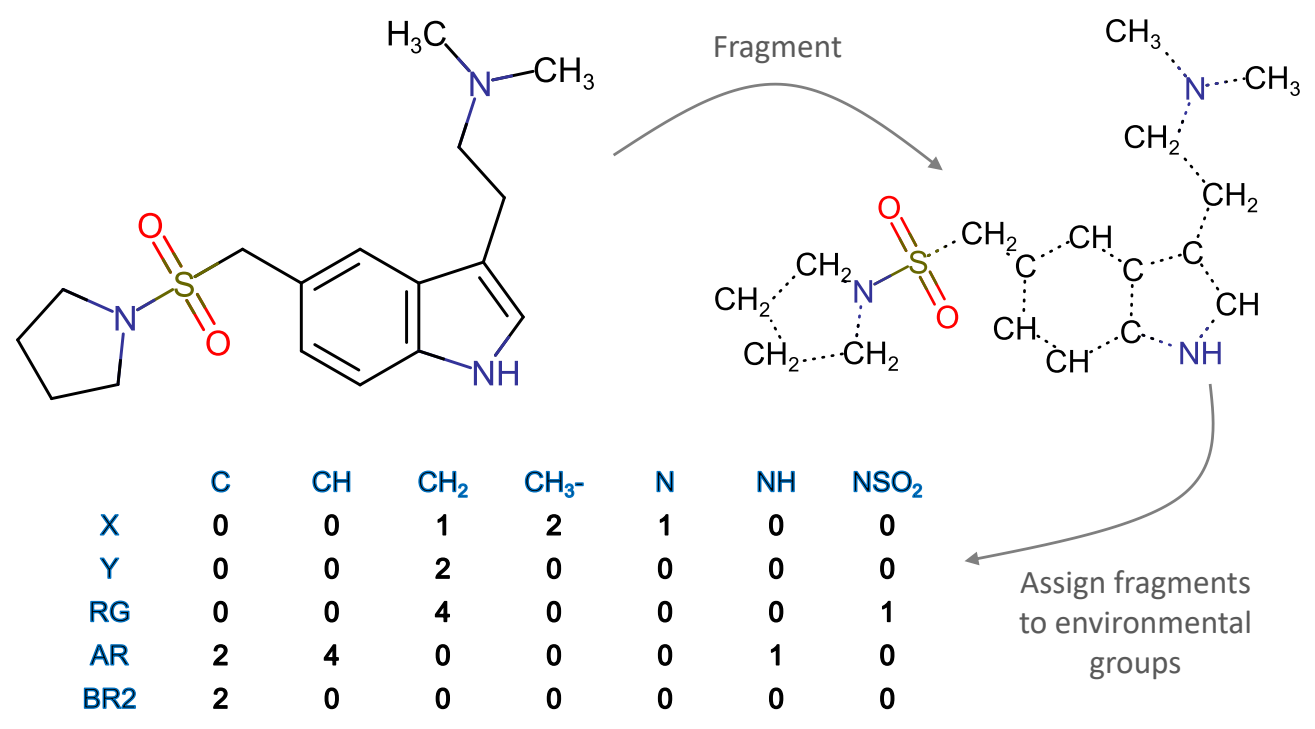

Figure 1: 2D structure of almotriptan molecule fragmented according to the isolating carbon method. Dashed lines represent broken bonds. Fragments (actually labeled by SMARTS strings in the software to avoid ambiguity) are then assigned to environmental groups.

\section{Geometrical Descriptors}

Entropy depends on molecular geometry and encodes translational, conformational, and rotational changes of a molecule that affect properties such as packing efficiency and the likeliness for initial and final states of a phase transition to exist. Entropy is given by

$$
\Delta S_{t r}=\Delta S_{t r}^{\text {trans }}+\Delta S_{t r}^{\text {conf }}+\Delta S_{t r}^{r o t}
$$

Components of $\Delta S_{t r}$ are computed from geometrical descriptors (descriptions below): eccentricity $(\epsilon)$, flexibility $(\phi)$, and symmetry $(\sigma)$.

Eccentricity is computed as the sum of atoms in and directly attached to aromatic rings. It is a qualitative measure of the packing efficiency since flat molecules tend to have less than average free volume due to their efficient packing, requiring more energy and a higher temperature to melt. Conversely, crystals made up of spherical molecules pack less efficiently and are more prone to attaining their free rotation.

Flexibility is a measure of the internal conformational freedom of a molecule. Flexible 
molecules tend to have a greater entropy change during melting than rigid molecules. In UPPER, flexibility is calculated by an ad hoc expression uniting flexible segments,

$$
\phi=0.3 \mathrm{ROT}+\mathrm{LINSP} 3+0.5(\mathrm{BRSP} 3+\mathrm{SP} 2+\mathrm{RING})-1
$$

where LINSP3 is the number of nonring, nonterminal, nonbranched $s p^{3}$ atoms, ROT is the extra entropy produced by freely rotating $s p^{3}$ atoms and is calculated as ROT $=$ LINSP3 -4 , BRSP3 is the total number of nonring, nonterminal, branched $s p^{3}$ atoms, SP2 is the number of nonring, nonterminal $s p^{2}$ atoms, RING is the number of single, fused, or conjugated ring systems. Compounds with negative $\phi$ computed using Eq. 4 are assigned $\phi$ equal to zero.

Symmetry affects entropy and in particular the melting point. Symmetric molecules have a higher probability of being in the right orientation for crystallization than nonsymmetrical molecules of roughly the same weight. As a result, they tend to have a lower entropy of melting and higher melting point. Here, the method to compute $\sigma$ (see Ref. [67]) operates by locating the center or centers of graphical symmetry and the equivalence classes of atoms connected to those centers. $\sigma$ is estimated based on a few simple rules determined by the hybridization of the graphical center as well as the number of connected atoms and their equivalence classes.

\section{UPPER-Inspired Fingerprint}

A combination of group-constitutive and geometrical descriptors make up what we call an UPPER-inspired fingerprint. It is single vector molecular representation, which can be used as input for machine learning techniques. The overall size of this fingerprint depends on the molecules in the dataset, as this affects the types of fragments and environmental groups (Table 1). Figure 2 shows example fingerprints for two molecules. 

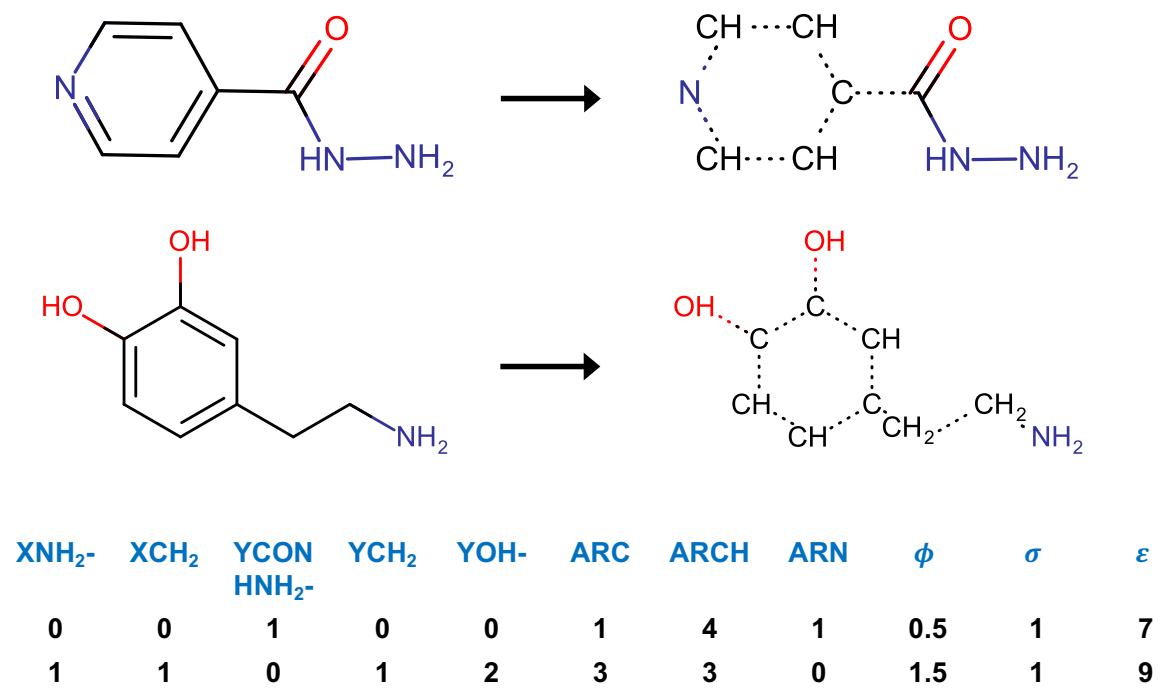

Figure 2: Isoniazid and dopamine molecules fragmented and their UPPER-inspired fingerprints consisting of group-constitutive and geometrical $(\phi, \sigma$, and $\epsilon)$ descriptors.

\section{Datasets and Learning Algorithms}

The dataset used in this work includes experimental transition enthalpies, entropies, and temperatures for both melting and boiling. Compounds of this dataset include open-chain, aliphatic, and aromatic compounds including polyhalogenated biphenyls, dibenzo-p-dioxins, diphenyl ethers, anisoles, and alkanes. There are a wide variety of functional groups such as alcohol, aldehyde, ketone, carboxylic acid, carbonate, carbamate, amine, amide, nitrile, acetyl, and nitro groups. The minimum, maximum, and mean number of atoms of the dataset are 2 (4), 42 (122), and 12 (24), where values in parentheses include hydrogen atoms. See Supporting Information showing a small subset of the compounds in this dataset as well as distributions of enthalpies, entropies, and temperatures. The exact dataset used is proprietary, supplied by an industrial collaborator of one of the authors. Thus, we cannot provide that particular dataset with this report. However, for the sake of transparency, another dataset that is very similar to the one used here is provided and described in Ref. [68]. This dataset has been collected and provided in our GitHub repository, allowing readers to reproduce our methods using the source code and data provided at the repository. 
In order to highlight usefulness of the proposed method of this work, two applications are carried out. The first is to predict melting points of energetic materials. Energetic materials must meet strict criteria to be developed and designated viable for applications. Knowledge of melting temperatures prior to synthesis would be particularly beneficial for the design of melt-casted explosives. 2 ${ }^{2}$ 4 A small dataset of energetic materials made up of $\sim 130$ melting points of nitroaliphatic and nitroaromatic compounds is added to the dataset. Finally, we carry out another application using large and diverse public datasets of melting points, labeled OCHEM, Enamine, Bradely, and Bergström. We refer readers to Refs. [69] and [20] for descriptions of these datasets. Both the energetic and public datasets are provided in the GitHub repository.

Calculations are carried out with two learning algorithms. UPPER is the reference model, where coefficients $p_{i}$ for enthalpy (Eq. 2) are determined using ridge regression. Ridge regression is a variant of linear regression with regularization to reduce overfitting. Although linear regression is actually used for Yalkowsky's UPPER, $[63$ ridge regression proves to be significantly better on the held-out test sets and thus will be used here as the standard. Entropies are also parameterized using ridge regression. The second model is a variant of Gradient Boosting (GB) called eXtreme GB or XGBoost. [65 A GB model is an ensemble of decision trees, where subsequent trees are trained to the residual error of the preceding tree. ${ }^{70}$ XGBoost controls overfitting better than GB, giving it strong performance. ${ }^{71}$ An added advantage is XGBoost's computational speed. ${ }^{[72}$ Ref. [72] compares XGBoost to deep neural networks for QSPRs and found that XGBoost achieved the same accuracy of neural networks with significantly less computational resources. XGBoost achieved its results on a single CPU, whereas efficient neural networks generally run on GPUs. This detail is an important practical advantage of XGBoost, especially in the laboratory setting with a standard workstation class computer. Although nonlinear machine learning generally provides more flexibility to learn the input data, it is important to note that the experimental datasets can be rather limited and noisy, greatly influencing a model's accuracy and transferability. 
Thus, the learning algorithms should be adequately assessed. Training details for the models including hyperparametrization are provided in Supporting Information.

\section{Results and Discussion}

We compare UPPER using ridge regression (UPPER-RR) and gradient boosting (UPPERGB). Models of enthalpy $\left(\Delta H_{t r}\right)$ and entropy $\left(\Delta S_{t r}\right)$ are compared across these two learning algorithms. Following enthalpy and entropy predictions, we compare transition temperatures $\left(T_{t r}\right)$, evaluated using Eq. 1. Our analysis suggests that gradient boosting does not provide significant improvement over ridge regression for enthalpies, entropies, and transition temperatures within the UPPER framework.

The main advance of this work is to use all group-constitutive and geometrical descriptors of UPPER and train directly to $T_{t r}$ with the GB algorithm, eliminating intermediate models for $\Delta H_{t r}$ and $\Delta S_{t r}$. We find this approach (ARL-UPPER) predicts $T_{t r}$ to greater accuracy than UPPER-RR and UPPER-GB. Finally, ARL-UPPER is applied to predict the melting points of energetic materials and large public datasets. Throughout this work, data are randomly split into $90 \%$ for training and $10 \%$ for testing. Prediction errors are quantified by the Root-Mean-Square-Error (RMSE) and the Mean-Absolute-Error (MAE).

\section{Enthalpy}

Fig. 3 shows parity plots of enthalpy of melting $\left(\Delta H_{m}\right)$ and boiling $\left(\Delta H_{b}\right)$. Only groupconstitutive descriptors (Table 1) were used in the models of Fig. 3. The results using UPPER-RR and UPPER-GB are comparable, showing that the nonlinear GB algorithm does not provide any added improvement in predicting $\Delta H_{t r}$ given group-constitutive descriptors. The prediction accuracy of $\Delta H_{m}$ is not as strong as that of $\Delta H_{b}$ likely due to missed intermolecular interactions in the crystal and liquid phases such as hydrogen bonding. These interactions are not as significant during a liquid-to-gas transition since molecules are more 
spatially separated. As a result, $\Delta H_{b}$ is predicted with greater accuracy than $\Delta H_{m}$ (i.e., RMSEs of $\sim 2$ compared to $\sim 4 \mathrm{~kJ} / \mathrm{mol}$ ) using knowledge of only a single molecular unit.
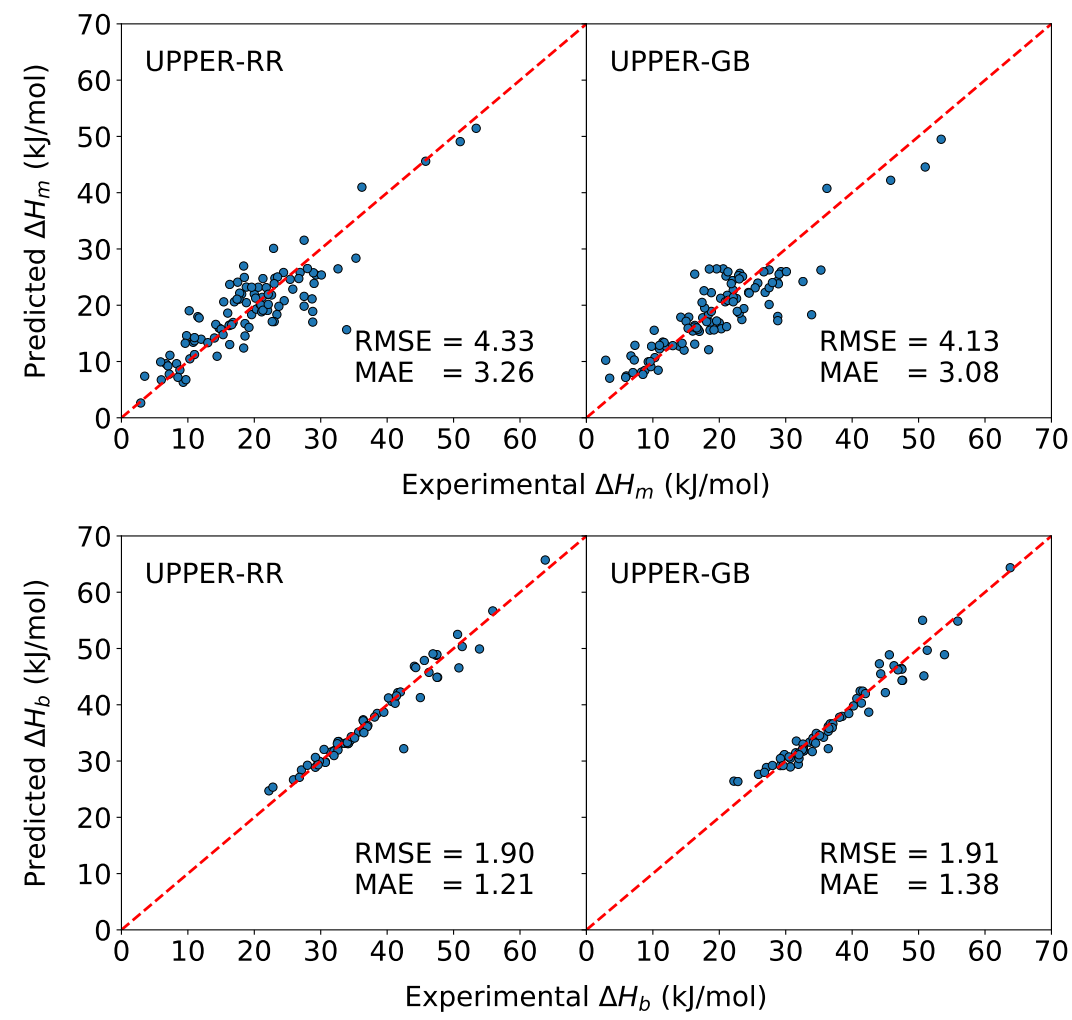

Figure 3: Parity plots of predicted versus experimental $\Delta H_{m}$ (top panels) and $\Delta H_{b}$ (bottom panels) using UPPER-RR (left panels) and UPPER-GB (right panels). Results are of the $10 \%$ held-out test set consisting of 108 and 68 molecules for $\Delta H_{m}$ and $\Delta H_{b}$, respectively. Prediction errors are shown in subpanels. Intermolecular interactions in the crystal-liquid phases (e.g., hydrogen bonding) are not completely accounted for with the group-constitutive descriptors. These missed interactions are likely the cause for the difference in prediction accuracy between $\Delta H_{m}$ and $\Delta H_{b}$.

\section{Entropy}

Parity plots of $\Delta S_{m}$ and $\Delta S_{b}$ are provided in Supporting Information. Similar to $\Delta H_{t r}$, $\Delta S_{t r}$ predictions are not significantly improved using GB. Trends in the entropy data can be explained by considering physical differences between melting and boiling. For the majority of the data, $\Delta S_{m}$ is smaller than $\Delta S_{b}$; a consequence of the relative change of molar volume during a crystal-to-liquid transition versus a liquid-to-gas transition. In particular, boiling produces a volumetric change of usually more than 20 liters per mol, whereas melting pro- 
duces a smaller change of a few cubic centimeters per mole. Further, $\Delta S_{b}$ data are clumped around $85-90 \mathrm{~J} / \mathrm{mol} . \mathrm{K}$ (Trouton's rule $]^{1}$, whereas $\Delta S_{m}$ shows more variability with a few compounds in the $150-350 \mathrm{~J} / \mathrm{mol} . \mathrm{K}$ range. The high $\Delta S_{m}$ compounds are long chain-like structures with single bonds that tend to orient themselves in parallel fashion to achieve maximum dispersion in the crystal phase (see Supporting Information). In the liquid phase, their flexible segments have a high degree of conformational freedom. Cross-validation results of $\Delta H_{t r}$ and $\Delta S_{t r}$ are provided in Supporting Information.

\section{Transition Temperatures}

Given trained models of $\Delta H_{t r}$ and $\Delta S_{t r}$, the ratio of their predictions (Eq. 11) gives $T_{t r}$ (Fig. 4).2 UPPER-GB slightly outperforms UPPER-RR, but the overall prediction error is still quite high (RMSEs of $45-55 \mathrm{~K}$ ). This result raises the question whether indirectly training to $T_{t r}$ and enforcing Eq. 1 impedes the model's predictive ability. Constructing the ARL-UPPER model, we may feed the entire set of group-constitutive and geometrical descriptors into the GB algorithm and train the model directly to $T_{t r}$. In this way, Eq. 1 is not directly enforced, allowing the learning algorithm to choose respective weights over its input features. The same 90-10 split is used here. The parity plots of Fig. 5 suggest that this flexibility is important as RMSEs decrease by about $20 \mathrm{~K}$ for $T_{m}$ and $T_{b}$. For reliable evaluation, averaged results ( \pm one standard deviation) over a 10 -fold cross-validation are provided in Table 2. To identify the added benefit of using a combination of group-constitutive and geometrical descriptors, models were trained solely to group-constitutive descriptors, resulting in slightly increased RMSEs (Table 2).

ARL-UPPER provides a systematic way of assessing new descriptors. For example, Ref. [73] relates molecular mass $(m)$ to $T_{m}$ using an expression for atomic vibrations in a monatomic solid in a thermal environment. The use of $m$ as a descriptor has been shown

\footnotetext{
${ }^{1}$ Trouton's rule states that the ratio of the volume of an organic compound as a gas to its volume as a liquid is constant at about $84 \mathrm{~J} / \mathrm{mol} . \mathrm{K}$.

${ }^{2}$ Due to limited experimental data, $\Delta H_{t r}$ and $\Delta S_{t r}$ models were trained to all available data. Therefore, results of Fig. 4 are likely biased.
} 

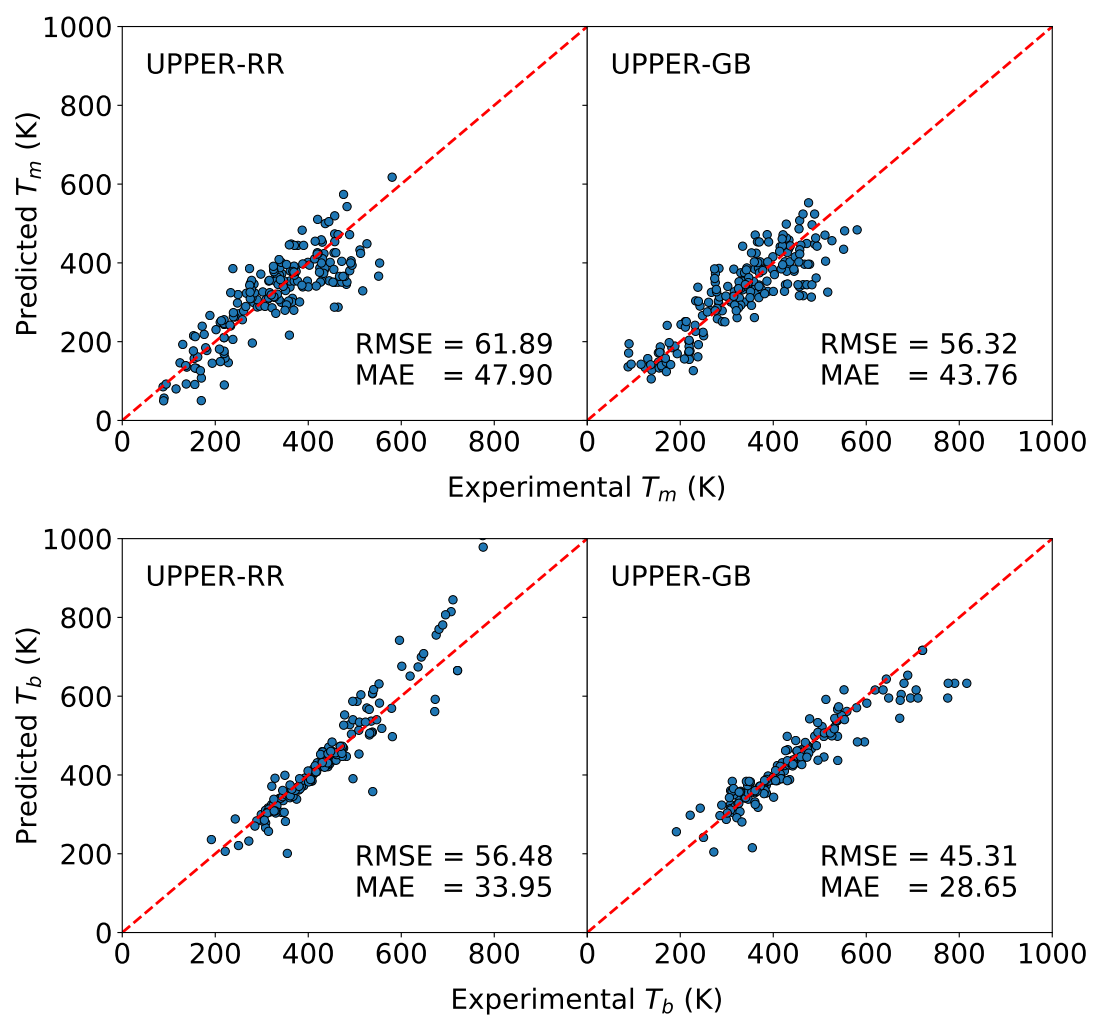

Figure 4: Parity plots of predicted versus experimental $T_{m}$ (top panels) and $T_{b}$ (bottom panels) using UPPER-RR (left panels) and UPPER-GB (right panels). Trained models of $\Delta H_{t r}$ and $\Delta S_{t r}$ were used to supply predictions of $\Delta H_{t r}$ and $\Delta S_{t r}$ to compute $T_{t r}$ (Eq,1). Test results consist of 202 and 168 molecules for $T_{m}$ and $T_{b}$, respectively. Prediction errors are shown in subpanels. Despite clear correlations, prediction errors are rather high and could use improvement.

to improve $T_{m}$ predictions. 17 Indeed, we find that the cross-validation RMSE of $T_{m}$ reduces slightly (Table 2). Significant improvement of $\sim 5 \mathrm{~K}$ is observed in the case of $T_{b}$. Lighter molecules have greater thermal motion than heavier molecules with the same kinetic energy. Thus, lighter molecules boil at lower temperatures, justifying the strong dependence of $T_{b}$ on $m$.

The results of ARL-UPPER are certainly encouraging (Table 2). However, it is important to acknowledge weaknesses of the approach. For example, the method's descriptors inadequately represent certain compounds. In particular, an example in Supporting Information shows structurally similar compounds with different $T_{m}$. Each molecule has an anthracene substructure functionalized by a methyl group, differing only by the methyl's position. Their UPPER-inspired fingerprints are the same, yet $T_{m}$ of 2-methylanthracene 

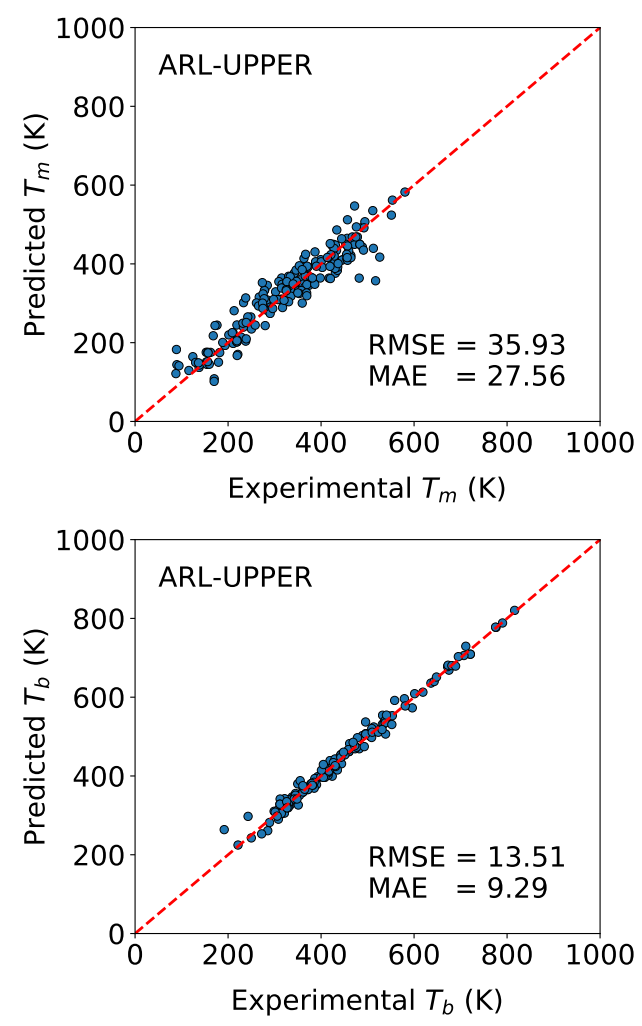

Figure 5: Parity plots of directly predicted $T_{m}$ (top panel) and $T_{b}$ (bottom panel) values using ARL-UPPER vs. experiment. Results are of the 10\% held-out test sets consisting of 202 and 168 molecules for $T_{m}$ and $T_{b}$, respectively. Prediction errors are shown in subpanels. Compared to Fig. 4. ARL-UPPER shows significant improvement in predicting $T_{t r}$.

is much larger than 1- and 9-methylanthracene. This significant difference is likely the result of packing arrangement. Unfortunately, packing is difficult to predict from molecular shape, especially when only using the $2 \mathrm{D}$ structure. We attempted replacing the current $2 \mathrm{D}$ eccentricity descriptor with $3 \mathrm{D}$ descriptors of eccentricity $\left(\epsilon_{3 D}\right)$ and asphericity $\left(q_{3 D}\right) .74$ Cross-validation predictions are slightly improved (Table 2), but while these conformational descriptors help distinguish the methylanthracene compounds, the added information is not sufficient enough for the model to map to their correct melting points. Thus, new descriptors encoding the effect of molecular shape on intermolecular interactions and subsequent expansion that occurs during melting are needed. The Wiener index-famous for its ability to encode topological information and its strong connection to boiling points of alkanes ${ }^{[75}$-was also tested as a descriptor $(w)$. Prediction errors further improved (Table 2), but overcoming 
Table 2: Prediction errors (RMSEs) of $T_{m}, T_{b}$ averaged over the 10-fold cross-validation test sets ( \pm one standard deviation). Models were trained to combinations of group-constitutive $(g c)$, geometrical $(g e o)$, and mass $(m)$ descriptors. Significant improvement in predictive ability is observed by directly training to $T_{t r}$ (ARL-UPPER) and adding descriptors that map to $T_{t r}$.

\begin{tabular}{c|c||c} 
Method & Descriptors & $T_{m}, T_{b}$ \\
\hline \hline UPPER-RR & $\Delta H_{t r}(g c), \Delta S_{t r}(g e o)$ & $62.9 \pm 4.1,59.2 \pm 6.8$ \\
\hline UPPER-GB & $\Delta H_{t r}(g c), \Delta S_{t r}(g e o)$ & $59.2 \pm 5.9,44.2 \pm 5.5$ \\
\hline \hline ARL-UPPER & $g c$ & $37.9 \pm 3.4,22.3 \pm 3.4$ \\
\hline ARL-UPPER & $g c, g e o$ & $36.0 \pm 3.5,21.8 \pm 4.4$ \\
\hline ARL-UPPER & $g c, g e o, m$ & $34.7 \pm 2.6,19.5 \pm 5.9$ \\
\hline ARL-UPPER & $g c, g e o\left(\epsilon_{3 D}, q_{3 D}\right), m$ & $31.4 \pm 2.5,19.3 \pm 4.6$ \\
\hline ARL-UPPER & $g c, g e o\left(\epsilon_{3 D}, q_{3 D}\right), m, w$ & $30.6 \pm 2.5,17.2 \pm 3.9$
\end{tabular}

the indistinguishability of the methylanthracene compounds remains a challenge (Supporting Information). Quantum-chemical prediction of crystal density [76] relates to packing and may provide useful information. Besides packing, the symmetry descriptor ${ }^{67}$ warrants improvement and is an opportunity for future research, as it does not distinguish stereoisomers such as cis-trans.

\section{Melting Points of Energetic Materials}

Fig. 6 shows an application of ARL-UPPER to predict $T_{m}$ of energetic materials containing many nitro groups. The descriptors of the model include all those used to produce the best cross-validation predictions of Table 2 (i.e., gc, geo $\left(\epsilon_{3 D}, q_{3 D}\right), m$, and $\left.w\right)$. The melting points reference dataset of the preceding subsection was augmented with energetics, making up about $5 \%$ of the entire training set ( $\sim 130$ compounds). The test set is a diverse set of nitroaliphatic and nitroaromatic compounds including nitropyrimidines and nitropyridines. Prediction accuracies are experimentally informative with overall test set RMSE of $35 \mathrm{~K}$. The model is particularly strong in the case of nitroaliphatic compounds with a RMSE of $25 \mathrm{~K}$. These results give promise toward predictive ML models of exotic energetics given comprehensive datasets. 


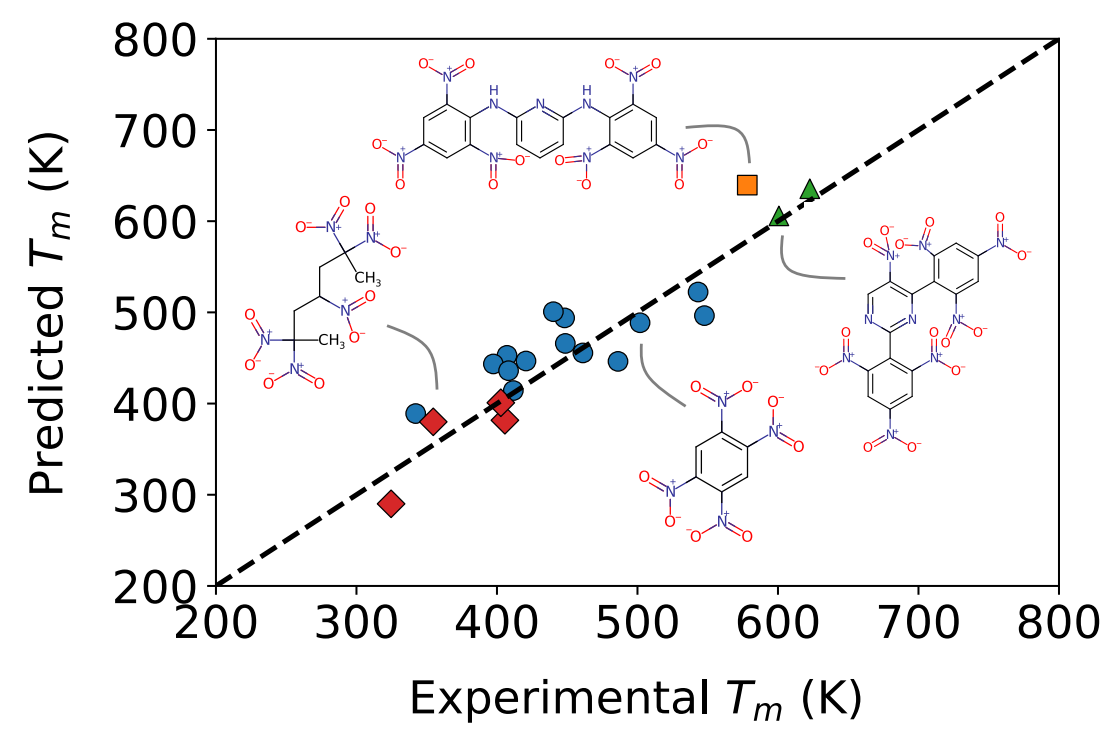

Figure 6: Parity plots of predicted versus experimental $T_{m}$ of energetic materials using ARLUPPER. Test set includes nitroaliphatics $(\diamond)$, nitroaromatics $(\bigcirc)$, nitropyridines $(\square)$, and nitropyrimidines $(\triangle)$.

\section{Melting Points of Public Datasets}

Finally, we apply ARL-UPPER to train and test on public datasets of $T_{m}$ (i.e., OCHEM, Enamine, Bradley, and Bergström). Due to their relatively small sizes and comparable temperature ranges, Bradley and Bergström are combined into one dataset, labeled BradBerg. Further details of the datasets can be found in Refs. [69] and [20]. Separate models were trained and tested on each dataset, again using the same 90-10 split. The top panel of Fig. 7 shows prediction error as a function of temperature for each of the datasets, while the bottom panel shows the distribution of temperatures. Not surprisingly, smallest errors are obtained for temperatures that make up the majority of the data. Table 3 reports cross-validation errors over the middle $50 \%$ and $323.15-523.15 \mathrm{~K}$; the latter being a popular range for medicinal compounds. ${ }^{[6]}$ The 30-40 K RMSE performance is very encouraging given the diversity of these datasets.

The datasets used for this application are unique in that they are both large and diverse 

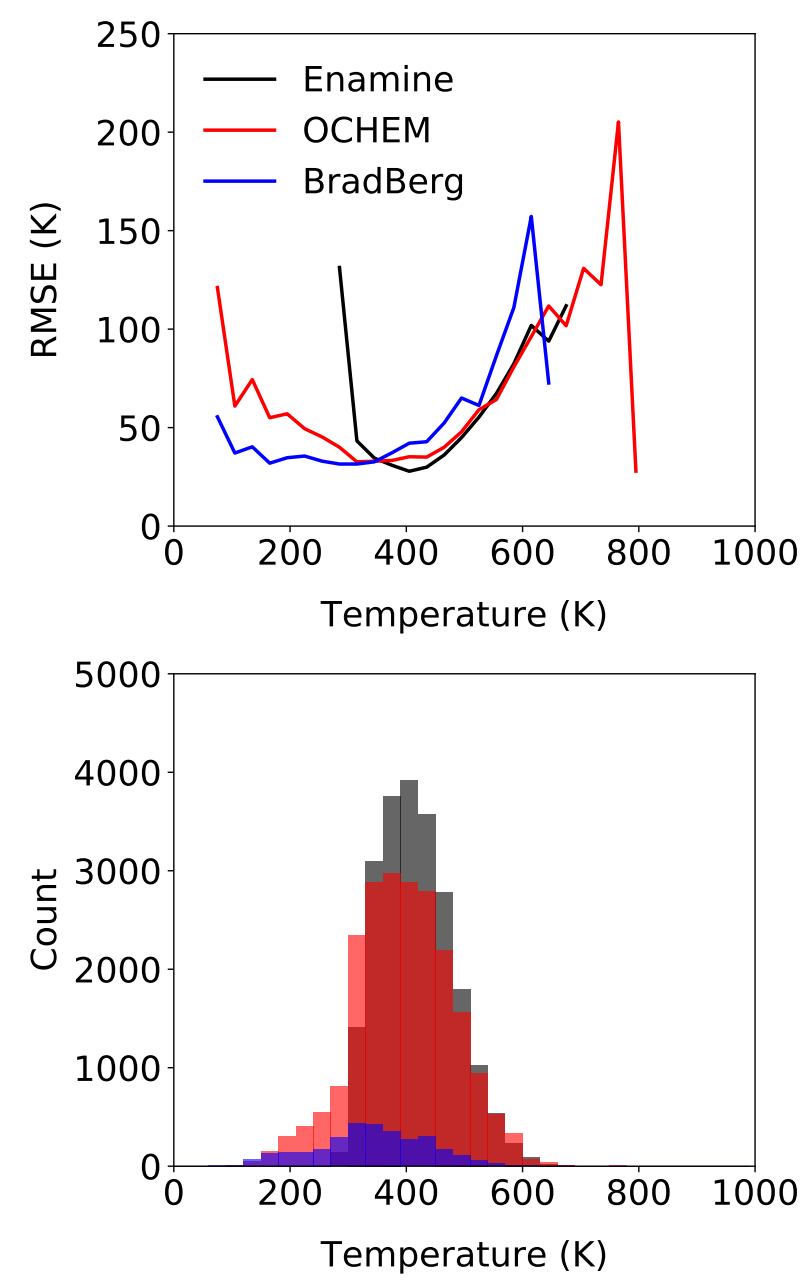

Figure 7: ARL-UPPER prediction errors (RMSEs) as a function of melting temperature for each dataset (top panel). Models were trained and tested on each dataset separately. Distributions of melting points for each dataset (bottom panel). Temperatures are binned every $30 \mathrm{~K}$. Performance correlates to the density of available data.

and can therefore be used for further curation, which would likely benefit applications targeting a specific chemical space. A model's applicable chemical space and accuracy are largely determined by the training dataset. 69 Thus, building a robust model is heavily dependent on the use of sufficient and high-quality data. Future work may benefit from advanced sampling techniques such as active learning; a semi-supervised procedure for data generation that interactively queries from a large dataset. ${ }^{77}$ As opposed to merely increasing the size of the dataset, active learning finds the right data for the task. During the curation process, it is also important to be mindful of sources of error such as experimental conditions and 
human error as trained models cannot overcome this irreducible error. It is worth noting that the Bradley and Bergström datasets are highly curated datasets of melting points and are considered the "gold standards".

Table 3: ARL-UPPER prediction errors (RMSEs, MAEs) of $T_{m}$ over the 10-fold crossvalidation test sets for the middle $50 \%$ and $323.15-523.15 \mathrm{~K}$ ranges.

\begin{tabular}{c||c|c|c} 
Dataset & Size & $T_{m}$, middle 50\% & $T_{m}, 323.15-523.15 \mathrm{~K}$ \\
\hline \hline Enamine & 22381 & $29.7,22.9$ & $34.1,26.3$ \\
\hline OCHEM & 21840 & $34.4,25.7$ & $37.3,27.7$ \\
\hline BradBerg & 3161 & $34.2,25.3$ & $41.8,30.9$
\end{tabular}

\section{Conclusion}

We presented a method that shows significant promise toward predicting experimental melting and boiling points of molecular materials. The descriptors originate from a linear regression based QSPR developed by Yalkowsky and coworkers known as UPPER (see Ref. [63]). UPPER's descriptors are primarily categorized as group-constitutive or geometrical, which model enthalpy and entropy; two thermodynamic quantities that drive thermal phase transitions. A notable advantage of UPPER's descriptors is that they are derived purely from SMILES string. Thus, besides simple structural characteristics such as connectivity and hybridization, there are no numerically intensive calculations necessary. This attribute of the method differs from other molecular representations that use expensive quantum mechanical calculations. 20169 In this work, we generalized UPPER to a more diverse chemical space by including descriptors that capture fragments with several $s p^{2}$-hybridized bonds. We also showed that an UPPER-inspired molecular representation, consisting of group-constitutive and geometrical descriptors, combined with eXtreme Gradient Boosting (denoted ARL-UPPER) produces highly competitive transition temperature predictions compared to the best results from the literature. 20169 Open-source software of our work is available at https://github.com/USArmyResearchLab/ARL-UPPER. 
Cross-validation RMSEs of melting and boiling points were found to be 36 and $20 \mathrm{~K}$, respectively (Table 2). Meanwhile, the dataset ranged from about $90-700 \mathrm{~K}$ and $150-850 \mathrm{~K}$. The model improved (reducing RMSEs to 31 and $17 \mathrm{~K}$ ) with mass, 3D descriptors of eccentricity and asphericity, and topological information using the Wiener index. ARL-UPPER also provided experimentally informative prediction of melting temperatures of energetic materials, highlighting its transferability to materials containing a significant number of nitro groups (compared to the majority of compounds used for training). The proposed method also achieved strong prediction errors within 30-40 K on melting points of large diverse public datasets. Its easy-to-compute descriptors, in combination with the accuracy and computational efficiency of XGBoost, makes ARL-UPPER a practical tool for screening transition temperatures in a laboratory setting.

This work has inspired other projects such as how the present method compares to common molecular fingerprints (see Supporting Information showing ARL-UPPER outperforming MACCS or Molecular ACCess System ${ }^{[78}$ structural keys using XGBoost). This task goes hand-in-hand with thorough evaluation of fingerprints across learning algorithms. Additionally, the intelligent sampling of a training set and more robust descriptors of hydrogen bonding ${ }^{[79]}$ and polymorphs ${ }^{[80}$ could further improve the model's performance. In its original form, UPPER is a comprehensive QSPR, combining structural information to physicochem-

ical properties including heat of sublimation, solubility, and vapor pressure. Furthering this work could make the ARL-UPPER framework a user-friendly screening tool for the design and discovery of materials in chemistry, physics, and materials science.

\section{Supporting Information Available}

Dataset information, training details, model predictions of phase transition properties including transition enthalpies, entropies, temperatures, and an example calculation of the ARL-UPPER fingerprint. Our software and datasets are freely available at 
https://github.com/USArmyResearchLab/ARL-UPPER

\section{Acknowledgement}

Research was sponsored by the U.S. Army Research Laboratory and was accomplished under Cooperative Agreement Number W911NF-19-2-0090. The views and conclusions contained in this document are those of the authors and should not be interpreted as representing the official policies, either expressed or implied, of the U.S. Army Research Laboratory or the U.S. Government. The U.S. Government is authorized to reproduce and distribute reprints for Government purposes notwithstanding any copyright notation. This work was supported in part by a grant of computer time from the DOD High Performance Computing Modernization Program at the ARL DoD Supercomputing Resource Center. We thank Brendan Gifford and Jason Morrill for fruitful discussions.

\section{References}

(1) Ran, Y.; Yalkowsky, S. H. Prediction of Drug Solubility by the General Solubility Equation (GSE). J. Chem. Inf. Comput. Sci. 2001, 41, 354-357.

(2) Ravi, P.; Badgujar, D. M.; Gore, G. M.; Tewari, S. P.; Sikder, A. K. Review on Melt Cast Explosives. Propellants Explos. Pyrotech. 2011, 36, 393-403.

(3) Johnson, E. C.; Sabatini, J. J.; Chavez, D. E.; Sausa, R. C.; Byrd, E. F.; Wingard, L. A.; Guzmàn, P. E. Bis (1, 2, 4-oxadiazole) bis (methylene) Dinitrate: A High-Energy MeltCastable Explosive and Energetic Propellant Plasticizing Ingredient. Org. Process Res. Dev. 2018, 22, 736-740.

(4) Johnson, E. C.; Bukowski, E. J.; Sabatini, J. J.; Sausa, R. C.; Byrd, E. F.; Garner, M. A.; Chavez, D. E. Bis (1, 2, 4-oxadiazolyl) Furoxan: A Promising Melt-Castable Eutectic Material of Low Sensitivity. ChemPlusChem 2019, 84, 319-322. 
(5) Zalba, B.; Marm, J. M.; Cabeza, L. F.; Mehling, H. Review on Thermal Energy Storage with Phase Change: Materials, Heat Transfer Analysis and Applications. Appl. Therm. Eng. 2003, 23, 251-283.

(6) Sharma, A.; Tyagi, V. V.; Chen, C.; Buddhi, D. Review on Thermal Energy Storage with Phase Change Materials and Applications. Renew. Sustain. Energy Rev. 2009, 13, 318-345.

(7) Agrawal, P. M.; Rice, B. M.; Thompson, D. L. Molecular Dynamics Study of the Melting of Nitromethane. J. Chem. Phys. 2003, 119, 9617-9627.

(8) Zhang, Y.; Maginn, E. J. A Comparison of Methods for Melting Point Calculation using Molecular Dynamics Simulations. J. Chem. Phys. 2012, 136, 144116.

(9) Brorsen, K. R.; Willow, S. Y.; Xantheas, S. S.; Gordon, M. S. The Melting Temperature of Liquid Water with the Effective Fragment Potential. J. Phys. Chem. Lett. 2015, 6, $3555-3559$.

(10) Chen, L.; Bryantsev, V. S. A Density Functional Theory Based Approach for Predicting Melting Points of Ionic Liquids. Phys. Chem. Chem. Phys. 2017, 19, 4114-4124.

(11) Katritzky, A. R.; Lomaka, A.; Petrukhin, R.; Jain, R.; Karelson, M.; Visser, A. E.; Rogers, R. D. QSPR Correlation of the Melting Point for Pyridinium Bromides, Potential Ionic Liquids. J. Chem. Inf. Comput. Sci. 2002, 42, 71-74.

(12) Dearden, J. C. Quantitative Structure-Property Relationships for Prediction of Boiling Point, Vapor Pressure, and Melting Point. Environ. Toxicol. Chem. 2003, 22, 16961709 .

(13) Trohalaki, S.; Pachter, R.; Drake, G. W.; Hawkins, T. Quantitative Structure-Property Relationships for Melting Points and Densities of Ionic Liquids. Energy 83 Fuels 2005, 19, 279-284. 
(14) Yuan, W.; Hansen, A. C.; Zhang, Q. Vapor Pressure and Normal Boiling Point Predictions for Pure Methyl Esters and Biodiesel Fuels. Fuel 2005, 84, 943-950.

(15) Preiss, U. P.; Beichel, W.; Erle, A. M.; Paulechka, Y. U.; Krossing, I. Is Universal, Simple Melting Point Prediction Possible? ChemPhysChem 2011, 12, 2959-2972.

(16) Morrill, J. A.; Byrd, E. F. Development of Quantitative Structure Property Relationships for Predicting the Melting Point of Energetic Materials. J. Mol. Graph. Model. 2015, 62, 190-201.

(17) Godavarthy, S. S.; Robinson, R. L.; Gasem, K. A. An Improved Structure-Property Model for Predicting Melting-Point Temperatures. Ind. Eng. Chem. Res. 2006, 45, $5117-5126$.

(18) Varnek, A.; Kireeva, N.; Tetko, I. V.; Baskin, I. I.; Solov'ev, V. P. Exhaustive QSPR Studies of a Large Diverse Set of Ionic Liquids: How Accurately Can We Predict Melting Points? J. Chem. Inf. Model. 2007, 47, 1111-1122.

(19) Seko, A.; Maekawa, T.; Tsuda, K.; Tanaka, I. Machine Learning with Systematic Density-Functional Theory Calculations: Application to Melting Temperatures of Single-and Binary-Component Solids. Phys. Rev. B 2014, 89, 054303.

(20) Jackson, N.; Sanchez-Lengeling, B.; Vazquez-Mayagoitia, A.; AspuruGuzik, A.; Vishwanath, V.; de Pablo, J. A Diversified Machine Learning Strategy for Predicting and Understanding Molecular Melting Points. 2019; https://chemrxiv.org/articles/A_Diversified_Machine_Learning_Strategy_ for_Predicting_and_Understanding_Molecular_Melting_Points/9914378.

(21) Smith, J. S.; Isayev, O.; Roitberg, A. E. ANI-1: An Extensible Neural Network Potential with DFT Accuracy at Force Field Computational Cost. Chem. Sci. 2017, 8, 31923203. 
(22) Nebgen, B.; Lubbers, N.; Smith, J. S.; Sifain, A. E.; Lokhov, A.; Isayev, O.; Roitberg, A. E.; Barros, K.; Tretiak, S. Transferable Dynamic Molecular Charge Assignment using Deep Neural Networks. J. Chem. Theory Comput. 2018, 14, 4687-4698.

(23) Sifain, A. E.; Lubbers, N.; Nebgen, B. T.; Smith, J. S.; Lokhov, A. Y.; Isayev, O.; Roitberg, A. E.; Barros, K.; Tretiak, S. Discovering a Transferable Charge Assignment Model using Machine Learning. J. Phys. Chem. Lett. 2018, 9, 4495-4501.

(24) Butler, K. T.; Davies, D. W.; Cartwright, H.; Isayev, O.; Walsh, A. Machine Learning for Molecular and Materials Science. Nature 2018, 559, 547.

(25) Hansen, K.; Biegler, F.; Ramakrishnan, R.; Pronobis, W.; Von Lilienfeld, O. A.; Müller, K.-R.; Tkatchenko, A. Machine Learning Predictions of Molecular Properties: Accurate Many-Body Potentials and Nonlocality in Chemical Space. J. Phys. Chem. Lett. 2015, 6, 2326-2331.

(26) Chmiela, S.; Tkatchenko, A.; Sauceda, H. E.; Poltavsky, I.; Schütt, K. T.; Müller, K.R. Machine Learning of Accurate Energy-Conserving Molecular Force Fields. Sci. Adv. 2017, 3, e1603015.

(27) Bleiziffer, P.; Schaller, K.; Riniker, S. Machine Learning of Partial Charges Derived from High-Quality Quantum-Mechanical Calculations. J. Chem. Inf. Model. 2018, 58, $579-590$.

(28) Lubbers, N.; Smith, J. S.; Barros, K. Hierarchical Modeling of Molecular Energies using a Deep Neural Network. J. Chem. Phys. 2018, 148, 241715.

(29) Li, H.; Collins, C.; Tanha, M.; Gordon, G. J.; Yaron, D. J. A Density Functional Tight Binding Layer for Deep Learning of Chemical Hamiltonians. J. Chem. Theory Comput. 2018, 14, 5764-5776. 
(30) St John, P.; Guan, Y.; Kim, Y.; Kim, S.; Paton, R. Prediction of Homolytic Bond Dissociation Enthalpies for Organic Molecules at Near Chemical Accuracy with SubSecond Computational Cost. 2019; https://chemrxiv.org/articles/Prediction_ of_Homolytic_Bond_Dissociation_Enthalpies_for_Organic_Molecules_at_ near_Chemical_Accuracy_with_Sub-Second_Computational_Cost/10052048.

(31) Smith, J. S.; Nebgen, B. T.; Zubatyuk, R.; Lubbers, N.; Devereux, C.; Barros, K.; Tretiak, S.; Isayev, O.; Roitberg, A. E. Approaching Coupled Cluster Accuracy with a General-Purpose Neural Network Potential Through Transfer Learning. Nat. Commun. 2019, 10, 2903.

(32) Ye, S.; Hu, W.; Li, X.; Zhang, J.; Zhong, K.; Zhang, G.; Luo, Y.; Mukamel, S.; Jiang, J. A Neural Network Protocol for Electronic Excitations of N-methylacetamide. Proc. Natl. Acad. Sci. 2019, 116, 11612-11617.

(33) Stein, H. S.; Guevarra, D.; Newhouse, P. F.; Soedarmadji, E.; Gregoire, J. M. Machine Learning of Optical Properties of Materials-Predicting Spectra from Images and Images from Spectra. Chem. Sci. 2019, 10, 47-55.

(34) Ghosh, K.; Stuke, A.; Todorović, M.; Jørgensen, P. B.; Schmidt, M. N.; Vehtari, A.; Rinke, P. Deep Learning Spectroscopy: Neural Networks for Molecular Excitation Spectra. Adv. Sci. 2019, 6, 1801367.

(35) Hu, W.; Ye, S.; Zhang, Y.; Li, T.; Zhang, G.; Luo, Y.; Mukamel, S.; Jiang, J. Machine Learning Protocol for Surface Enhanced Raman Spectroscopy. J. Phys. Chem. Lett. 2019, 10, 6026-6031.

(36) Kananenka, A. A.; Yao, K.; Corcelli, S. A.; Skinner, J. L. Machine Learning for Vibrational Spectroscopic Maps. 2019; https://doi.org/10.1021/acs.jctc.9b00698.

(37) Raccuglia, P.; Elbert, K. C.; Adler, P. D.; Falk, C.; Wenny, M. B.; Mollo, A.; Zeller, M.; 
Friedler, S. A.; Schrier, J.; Norquist, A. J. Machine-Learning-Assisted Materials Discovery using Failed Experiments. Nature 2016, 533, 73.

(38) Coley, C. W.; Green, W. H.; Jensen, K. F. Machine Learning in Computer-Aided Synthesis Planning. Acc. Chem. Res. 2018, 51, 1281-1289.

(39) de Almeida, A. F.; Moreira, R.; Rodrigues, T. Synthetic Organic Chemistry Driven by Artificial Intelligence. Nat. Rev. Chem. 2019, 1-16.

(40) Li, Z.; Najeeb, M. A.; Alves, L.; Sherman, A.; Parrilla, P. C.; Pendleton, I. M.; Zeller, M.; Schrier, J.; Norquist, A. J.; Chan, E. Robot-Accelerated Perovskite Investigation and Discovery (RAPID): 1. Inverse Temperature Crystallization. 2019; https: //chemrxiv.org/articles/Robot-Accelerated_Perovskite_Investigation_and_ Discovery_RAPID_1_Inverse_Temperature_Crystallization/10013090.

(41) Fourches, D.; Muratov, E.; Tropsha, A. Trust, But Verify: On the Importance of Chemical Structure Curation in Cheminformatics and QSAR Modeling Research. J. Chem. Inf. Model. 2010, 50, 1189-1204.

(42) Mitchell, J. B. Machine Learning Methods in Chemoinformatics. Wiley Interdiscip. Rev. Comput. Mol. Sci. 2014, 4, 468-481.

(43) Zang, Q.; Mansouri, K.; Williams, A. J.; Judson, R. S.; Allen, D. G.; Casey, W. M.; Kleinstreuer, N. C. In Silico Prediction of Physicochemical Properties of Environmental Chemicals using Molecular Fingerprints and Machine Learning. J. Chem. Inf. Model. $\mathbf{2 0 1 7}, 57,36-49$.

(44) Seko, A.; Hayashi, H.; Nakayama, K.; Takahashi, A.; Tanaka, I. Representation of Compounds for Machine-Learning Prediction of Physical Properties. Phys. Rev. B 2017, 95,144110 . 
(45) Barnes, B. C.; Elton, D. C.; Boukouvalas, Z.; Taylor, D. E.; Mattson, W. D.; Fuge, M. D.; Chung, P. W. Machine Learning of Energetic Material Properties. 2018; https://arxiv.org/abs/1807.06156.

(46) Gómez-Bombarelli, R.; Wei, J. N.; Duvenaud, D.; Hernández-Lobato, J. M.; SánchezLengeling, B.; Sheberla, D.; Aguilera-Iparraguirre, J.; Hirzel, T. D.; Adams, R. P.; Aspuru-Guzik, A. Automatic Chemical Design Using a Data-Driven Continuous Representation of Molecules. ACS Cent. Sci. 2018, 4, 268-276.

(47) Vamathevan, J.; Clark, D.; Czodrowski, P.; Dunham, I.; Ferran, E.; Lee, G.; Li, B.; Madabhushi, A.; Shah, P.; Spitzer, M. et al. Applications of Machine Learning in Drug Discovery and Development. Nat. Rev. Drug Discov. 2019, 18, 463-477.

(48) Huang, B.; Von Lilienfeld, O. A. Communication: Understanding Molecular Representations in Machine Learning: The Role of Uniqueness and Target Similarity. J. Chem. Phys. 2016, 145, 161102.

(49) Collins, C. R.; Gordon, G. J.; Von Lilienfeld, O. A.; Yaron, D. J. Constant Size Descriptors for Accurate Machine Learning Models of Molecular Properties. J. Chem. Phys. 2018, $148,241718$.

(50) Behler, J. Perspective: Machine Learning Potentials for Atomistic Simulations. J. Chem. Phys. 2016, 145, 170901.

(51) Bartók, A. P.; De, S.; Poelking, C.; Bernstein, N.; Kermode, J. R.; Csányi, G.; Ceriotti, M. Machine Learning Unifies the Modeling of Materials and Molecules. Sci. Adv. 2017, 3, e1701816.

(52) Imbalzano, G.; Anelli, A.; Giofré, D.; Klees, S.; Behler, J.; Ceriotti, M. Automatic Selection of Atomic Fingerprints and Reference Configurations for Machine-Learning Potentials. J. Chem. Phys. 2018, 148, 241730. 
(53) Zhang, Y.; Hu, C.; Jiang, B. Embedded Atom Neural Network Potentials: Efficient and Accurate Machine Learning with a Physically Inspired Representation. J. Phys. Chem. Lett. 2019, 10, 4962-4967.

(54) Bergström, C. A.; Norinder, U.; Luthman, K.; Artursson, P. Molecular Descriptors Influencing Melting Point and Their Role in Classification of Solid Drugs. J. Chem. Inf. Comput. Sci. 2003, 43, 1177-1185.

(55) Ghiringhelli, L. M.; Vybiral, J.; Levchenko, S. V.; Draxl, C.; Scheffler, M. Big Data of Materials Science: Critical Role of the Descriptor. Phys. Rev. Lett. 2015, 114, 105503.

(56) Sun, Y.; Bai, H.; Li, M.; Wang, W. Machine learning approach for prediction and understanding of glass-forming ability. J. Phys. Chem. Lett. 2017, 8, 3434-3439.

(57) Elton, D. C.; Boukouvalas, Z.; Butrico, M. S.; Fuge, M. D.; Chung, P. W. Applying Machine Learning Techniques to Predict the Properties of Energetic Materials. Sci. Rep. 2018, 8, 9059.

(58) Brethomé, A. V.; Fletcher, S. P.; Paton, R. S. Conformational Effects on PhysicalOrganic Descriptors: The Case of Sterimol Steric Parameters. ACS Catalysis 2019, 9, 2313-2323.

(59) Cereto-Massagué, A.; Ojeda, M. J.; Valls, C.; Mulero, M.; Garcia-Vallvé, S.; Pujadas, G. Molecular Fingerprint Similarity Search in Virtual Screening. Methods 2015, 71, 58-63.

(60) Yap, C. W. PaDEL-Descriptor: An Open Source Software to Calculate Molecular Descriptors and Fingerprints. J. Comput. Chem. 2011, 32, 1466-1474.

(61) Lo, Y.-C.; Rensi, S. E.; Torng, W.; Altman, R. B. Machine Learning in Chemoinformatics and Drug Discovery. Drug Discov. Today 2018, 23, 1538-1546.

(62) Yang, K.; Swanson, K.; Jin, W.; Coley, C.; Eiden, P.; Gao, H.; Guzman-Perez, A.; 
Hopper, T.; Kelley, B.; Mathea, M. et al. Analyzing learned molecular representations for property prediction. J. Chem. Inf. Model. 2019, 59, 3370-3388.

(63) Lian, B.; Yalkowsky, S. H. Unified Physicochemical Property Estimation Relationships (UPPER). J. Pharm. Sci. 2014, 103, 2710-2723.

(64) Weininger, D. SMILES, A Chemical Language and Information System. 1. Introduction to Methodology and Encoding Rules. J. Chem. Inf. Comput. Sci. 1988, 28, 31-36.

(65) Chen, T.; Guestrin, C. Xgboost: A Scalable Tree Boosting System. Proceedings of the 22nd ACM SIGKDD International Conference on Knowledge Discovery and Data Mining. 2016; pp 785-794.

(66) Leo, A. J. Calculating Log P(oct) From Structures. Chem. Rev. 1993, 93, 1281-1306.

(67) Walters, W. P.; Yalkowsky, S. H. ESCHER A Computer Program for the Determination of External Rotational Symmetry Numbers from Molecular Topology. J. Chem. Inf. Comput. Sci. 1996, 36, 1015-1017.

(68) Jain, A.; Yalkowsky, S. H. Estimation of Melting Points of Organic Compounds-II. J. Pharm. Sci. 2006, 95, 2562-2618.

(69) Tetko, I. V.; Sushko, Y.; Novotarskyi, S.; Patiny, L.; Kondratov, I.; Petrenko, A. E.; Charochkina, L.; Asiri, A. M. How Accurately Can We Predict the Melting Points of Drug-Like Compounds? J. Chem. Inf. Model. 2014, 54, 3320-3329.

(70) Friedman, J.; Hastie, T.; Tibshirani, R. The Elements of Statistical Learning; Springer Series in Statistics, New York, 2001; Vol. 1.

(71) Feng, D.; Svetnik, V.; Liaw, A.; Pratola, M.; Sheridan, R. P. Building Quantitative Structure-Activity Relationship Models Using Bayesian Additive Regression Trees. J. Chem. Inf. Model. 2019, 59, 2642-2655. 
(72) Sheridan, R. P.; Wang, W. M.; Liaw, A.; Ma, J.; Gifford, E. M. Extreme Gradient Boosting as a Method for Quantitative Structure-Activity Relationships. J. Chem. Inf. Model. 2016, 56, 2353-2360.

(73) Austin, J. A Relation Between the Molecular Weights and Melting Points of Organic Compounds. J. Am. Chem. Soc. 1930, 52, 1049-1053.

(74) Todeschini, R.; Consonni, V. Handbook of Molecular Descriptors; John Wiley \& Sons, 2008; Vol. 11.

(75) Wiener, H. Structural Determination of Paraffin Boiling Points. J. Am. Chem. Soc. $1947,69,17-20$.

(76) Rice, B. M.; Byrd, E. F. Evaluation of Electrostatic Descriptors for Predicting Crystalline Density. J. Comput. Chem. 2013, 34, 2146-2151.

(77) Smith, J. S.; Nebgen, B.; Lubbers, N.; Isayev, O.; Roitberg, A. E. Less is More: Sampling Chemical Space with Active Learning. J. Chem. Phys. 2018, 148, 241733.

(78) Durant, J. L.; Leland, B. A.; Henry, D. R.; Nourse, J. G. Reoptimization of MDL Keys For Use in Drug Discovery. J. Chem. Inf. Model. 2002, 42, 1273-1280.

(79) Alantary, D.; Yalkowsky, S. H. Estimating the Physicochemical Properties of Polysubstituted Aromatic Compounds using UPPER. J. Pharm. Sci. 2018, 107, 297-306.

(80) Zhang, Y.; Maginn, E. J. Toward Fully in Silico Melting Point Prediction using Molecular Simulations. J. Chem. Theory Comput. 2013, 9, 1592-1599. 


\section{Graphical TOC Entry}

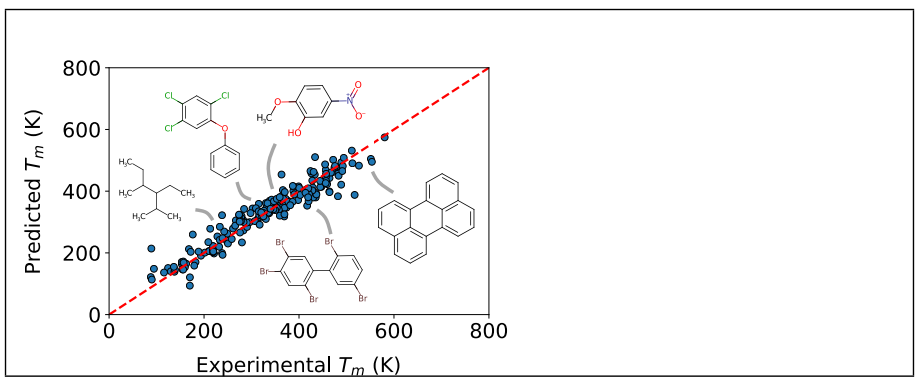

